\title{
Subjective zeros, subjectively equal stimulus spacing, and contraction biases in very first judgments of lightness
}

\author{
E. C. POULTON and D. C. V. SIMMONDS \\ Medical Research Council, Applied Psychology Unit, Cambridge, England
}

\begin{abstract}
Separate groups of 50 uninitiated students made (1) a very first partition judgment of a gray, reflectance 19\%, lying between light and dark standards, by marking a line; (2) a similar very first partition judgment with only a single standard; or (3) a very first fractional numerical judgment of lightness or darkness using the same display as (2). Comparing (2) with (1), a reflectance of 5\% corresponds to the intercept of zero judged lightness or black. A reflectance of $45 \%$ corresponds to zero judged darkness or white. Since (3) gives similar results to (2), these subjective zeros should apply also to the first fractional numerical judgments of lightness and darkness. Correcting for the subjective zeros, one unit of lightness corresponds to about the same reflectance ratio as one unit of darkness. The subjectively equal stimulus spacing for the very first partition judgments of lightness lies between logarithmic and the Munsell neutral value spacing, but closer to logarithmic. In marking a line for the first time, uninitiated students avoid both the middle and the ends of the line. The bias needs to be distinguished from the response contraction bias on regression effect, which may accompany it.
\end{abstract}

Reflectances extend theoretically from $0 \%$ to $100 \%$. But it is not possible to reach these limiting values without using some trick. Thus, what people ordinarily call black has a reflectance greater than $0 \%$. What people ordinarily call white has a reflectance less than $100 \%$. Suppose an observer is asked to judge how many times lighter or darker one shade of gray is than another shade of gray. In order to judge the ratio, the observer has to define a zero magnitude for gray, either explicitly or implicitly. The zero magnitude is here called the subjective zero.

The size of the subjective zero is important to those who follow S. S. Stevens (1975, Table I) and believe in an exponent of a characteristic size for each stimulus dimension. This is because finding a subjective zero, $\mathrm{S}_{0}$, appreciably above the physical zero reduces the size of the exponent by changing Stevens's power function for lightness from

$$
\mathbf{R}=\mathbf{k} \mathbf{S}^{\mathbf{n}}
$$

to

$$
\mathrm{R}=\mathrm{k}\left(\mathrm{S}-\mathrm{S}_{0}\right)^{\mathrm{n}}
$$

(Ekman, 1958). In these equations, $R$ stands for response and $\mathrm{S}$ for stimulus. The exponent, $\mathrm{n}$, is given by:

The authors are grateful to R. M. Warren for some useful comments and for recalibrating all the gray papers; they are also grateful to $\mathbf{R}$. J. Watt, A. J. Wilkins, and two anonymous referees for help with the reporting. Financial support from the British Medical Research Council is also gratefully acknowledged. Requests for reprints should be sent to E. C. Poulton, Medical Research Council, Applied Psychology Unit, 15 Chaucer Road, Cambridge, England CB2 2EF.

$$
\mathbf{n}=\frac{\log \text { geometric } \mathrm{R} \text { range }}{\log \text { geometric } \mathrm{S} \text { range }}
$$

(Teghtsoonian, 1971). Subtracting a constant $S_{0}$ from each value of $S$ increases the geometric stimulus range, and so reduces the size of the exponent.

By definition, the subjectively equal stimulus spacing is related linearly to the observers' responses when the responses are unbiased. Once the subjectively equal stimulus spacing is known, it can be used to indicate how observers use numbers. If the observers' responses are logarithmic when plotted against the subjectively equal stimulus spacing, numbers must be used as ratios (Birnbaum, 1978, 1980; Torgerson, 1960). If the observers' responses are intermediate between linear and logarithmic, numbers must be used with a logarithmic response bias.

The way the observers use numbers in making numerical magnitude judgments determines the appropriate method of deriving the subjective zero. If the observers use numbers linearly, their judgments are comparable to partition judgments. Thus, the subjective zero can be derived by the method used here for partition judgments. However, if the observers use numbers as ratios or with a logarithmic response bias, an alternative method is required that takes account of the nonlinearity in the responses.

The alternative method could use a set of first absolute judgments or of first fractional judgments made against a fixed standard, plot the mean judgments linearly against the stimuli, and extrapolate the curved function fitted to the means until it reaches the point where the subjective judgment would be zero. Here the stimulus value is the subjective zero, $S_{0}$. 
Unfortunately, in order to extrapolate accurately, unbiased points are needed not far above the subjective zero. Yet, the corresponding stimuli lie far from the middle of the range of possible stimuli. The first judgment may therefore be biased considerably by the response contraction bias (Poulton, 1979, p. 789) or regression effect (Stevens \& Greenbaum, 1966). The observers choose a response that lies too close to the middle of the range of responses. Thus, the method cannot be recommended for a first judgment.

In marking a line for the first time, the relative stimulus contraction bias describes the observers' avoidance of both the middle and the ends of the line (Poulton, 1985, chap. 7). Avoiding the middle of the line is reported by Poulton, Simmonds, and Warren (1968, Figure 1) for the first judgment of lightness. The complete bias is described by Taylor (1961) for plotting points on a rectangular card. The bias occurs in both the vertical and the horizontal dimensions. Observers tend to avoid both the middle and the edges of the card. Thus, the range of responses between the middle and the edges of the card is contracted. Hence the name "contraction bias." The bias is relative, because the students overestimate the small difference between the stimulus and what is presumably their reference point at the middle or edge of the card. The relative stimulus contraction bias may be accompanied by the response contraction bias or regression effect, which biases both first and subsequent judgments.

\section{Very First Judgments of Uninitiated Observers}

The investigation used the very first judgments of uninitiated observers because judgments after the first can be biased by prior judgments (Poulton, 1968, Figure 2; Warren \& Poulton, 1960, Table 1). It is sometimes assumed that the first judgment of an uninitiated observer is biased just like subsequent judgments. The only difference is that, for the first judgment, each uninitiated observer brings to the investigation his or her own prior context (Poulton, 1975, p. 6). This is presumably true. But with separate groups of perhaps 50 uninitiated observers, the average prior context of each group should be much the same, and should be representative of the population from which the observers are drawn. Thus, the prior context should be much the same for any investigation that uses large numbers of observers in each separate group, whereas the prior context of judgments after the first changes from one investigation to another, because it depends on the particular judgments made previously (Poulton, 1982).

\section{Subjective Zeros in Judging Lightness and Darkness}

Experiment 1 was designed to determine the subjective zeros for the first partition judgment of lightness and darkness, before the judgments were biased by prior context.

Figure 1 illustrates the method. Separate groups of uninitiated students made a first partition judgment of where a midgray with a reflectance of $19 \%$ lay between a light and a dark standard. They did so by marking a $100-\mathrm{mm}$ line labeled at the two ends to correspond to the two standards.

The subjective zero corresponding to black in judging lightness was determined by holding the white standard constant and varying the reflectance of the dark standard. This provided a function like that in Panel $\mathrm{A}$, where the reflectance of the dark standard is shown on the abscissa and is also printed against the B points. The ordinate gives the position of the average mark, measured from the end of the line that corresponds to black or dark gray.

An additional separate group made a similar judgment without a dark standard. The observers were presented with only the midgray and the white standard to judge it against. By entering the average position of the mark of the ordinate of Panel A and reading off from the graph, it is possible to determine the reflectance of the dark standard that would have given the same average response. This reflectance indicates the subjective zero for black in the absence of a dark standard, which is used by the average uninitiated student for the first partition judgment of lightness.

The subjective zero corresponding to white in judging darkness was determined by the complementary procedure. Here the dark standard was held constant and the light standard was varied. The additional separate group made a similar judgment without a light standard. The subjective zero at the white end was then read off a graph like that of Figure 1 Panel B.

\section{Correcting for the Subjective Zero in Fractional Numerical Judgments}

Experiment 2 was designed to determine whether the subjective zeros found for the first partition judgment in marking a line applied also to the first fractional numerical judgment. This was done by comparing the two kinds of judgment, using the same display. If both kinds of first judgment give statistically indistinguishable results, they presumably have the same subjective zero. If so, the subjective zero can be used to correct the ratio of the two reflectances selected for the first fractional numerical judgment. The corrected units for lightness and darkness should then be about the same size.

\section{Possible Subjectively Equal Stimulus Spacings for Lightness}

Partition judgments like those illustrated in Figure 1 can be compared with predictions derived from possible subjectively equal stimulus spacings. Substitute values corresponding to the possible subjectively equal stimulus spacing for the stimuli in the equation:

\section{Prediction}

$$
=\frac{\text { Midgray }- \text { Dark standard }}{\text { Light standard }- \text { Dark standard }} \times 100 .
$$

The most thoroughly researched subjectively equal stimulus spacing for the lightness of grays is the Munsell 
scale of neutral value (Newhall, Nickerson, \& Judd, 1943; see Warren, 1969, pp. 17-20). The Munsell neutral value can be described reasonably well by the equation:

$$
\text { Munsell neutral value }=\sqrt{\mathrm{Rf} \%}+\mathrm{K} \text {, }
$$

where Rf stands for reflectance (Longmore \& Petherbridge, 1961). The constant $\mathrm{K}$ is zero at $100 \%$ and at $0 \%$ reflectance. $\mathrm{K}$ has its maximum value of .61 from $22 \%$ to $15 \%$ reflectance.

Warren's $(1958,1969,1981)$ physical correlate theory predicts a subjectively equal stimulus spacing that is the square root of reflectance. A perhaps more obvious candidate is logarithmic spacing, log Rf, or nearly logarithmic spacing. This is the subjectively equal stimulus spacing found for partition judgments of brightness by Fagot, Eskildsen, and Stewart (1966). At the other extreme, the subjectively equal stimulus spacing could be linear in reflectance, although this seems unlikely.

\section{EXPERIMENT 1}

\section{Method}

Observers. The 602 observers were mainly men undergraduates of Cambridge University, although a few men graduates and women students were included. None had previously judged sensory magnitudes, and each student served in only one condition. Students of psychology were not used.

Display. A brown $70 \times 70 \mathrm{~cm}$ hardboard table top with a reflectance of $20 \%$ always served as a background. In the middle of it was placed a variable patch of paper, flanked on each side by a standard patch. The three patches each measured $28 \times 15 \mathrm{~cm}$. The patches lay together with their long sides contiguous and their short sides closest to the student. There were also conditions with only a single standard. For half the students in each condition, the light standard, or the only standard, was on the left. For the other half of the students, the display was reversed, with the light standard, or the only standard, on the right.

The white and gray patches consisted of paper coated with nonglossy paint, supplied by the Mitchell Colour Card Company, London. Black rayon velvet was also used. The reflectances were measured both with a Macbeth illuminometer and with an illuminometer made by Salford Electrical Instruments Limited. The lighting on the table was at the ambient level to which the students had become adapted. This appeared preferable to introducing a standard extraneous source of light. Previous investigations using separate groups of $\mathbf{5 0}$ students had shown that judgments of relative reflectance made in bright daylight were no different from judgments made in very much less bright artificial light.

Conditions. The conditions of Experiment 1 are listed on the left side of Table 1 . Column 4 shows that the midgray to judge always had a reflectance of $19 \%$. A reflectance of $19 \%$ was used because it lies close to the middle of the subjective range of reflectances between white and black. The Munsell scale of neutral value (Newhall et al., 1943, Table 2) extends from 10 to 0. On this scale, $19 \%$ reflectance has a value of 4.9 .

The reflectances of the light and dark standards on either side of the midgray are given in Columns 3 and 5 of Table 1 . The conditions in the top six rows of the table were used to determine the subjective zero corresponding to black in judging lightness. The constant light standard had the highest reflectance that was available, $83 \%$. The reflectance of the dark standard was varied between $.26 \%$ and $5.3 \%$.

The conditions in the bottom six rows of Table 1 were used to determine the subjective zero corresponding to white in judging darkness. The constant dark standard of $5.3 \%$ reflectance was the closest match to the subjective zero that was found to correspond to black in judging lightness. The reflectance of the light standard was varied between $83 \%$ and $44 \%$.

The reflectances of the light and dark standards and of the midgray were selected to keep the mean responses shown in Column 6 of Table 1 close to 50 , the middle of the available range of responses. This reduced the contraction biases, which increase in size as the mean response moves further from the middle of the available range of responses.

In Column 1 of Table 1 , the groups or conditions are labeled by the stimulus that distinguished them. Thus, Group B.26 had a black standard with a reflectance of $.26 \%$. Group B - had no black standard. Group W83 had a white standard with a reflectance of $83 \%$, and so on. Note that Group B5.3 is equivalent to Group W83.

Procedure. The investigation was conducted in Cambridge colleges. One investigator canvassed for volunteers and kept curious eyes off the display. When the student sat down at the table, the second investigator handed him or her a $20 \times 13 \mathrm{~cm}$ white card with instructions typed on it. Typical instructions read: "You will see three pieces of paper lying side by side, one black, one gray, and one white. You are to judge the lightness or darkness of the gray relative to the other two papers. A slip will be given you with a line marked 'black' at one end and 'white' at the other. Imagine this line to represent a scale of grays lying between black and white. Please place a mark on this line to indicate where you think the gray paper belongs on this scale."

When the light standard had a reflectance less than $83 \%$, it was described as "light gray" and the corresponding end of the response line was labeled "light gray." When the dark standard had a reflectance greater than $3.4 \%$, it was described as "dark gray" and the corresponding end of the response line was labeled "dark gray." When the light or dark standard was absent from the display, it was always described as "white" or "black," respectively, and the corresponding end of the response line was labeled "white" or "black." The first paragraph of the instructions then mentioned only two pieces of paper, the gray and a single standard.

After reading the instructions, the student was handed a pencil. A white $20 \times 13 \mathrm{~cm}$ piece of paper with a $100-\mathrm{mm}$ line drawn horizontally on it was placed on the edge of the hardboard table top in front of him or her. The line was labeled as described in the instructions and was oriented to match the display. Thus, when the light standard was on the left, the left end of the line was labeled to correspond to the light standard.

When no light standard was present in the display, the white response slip carrying the 100 -mm line was presented on a $33 \times$ $23 \mathrm{~cm}$ piece of brown hardboard similar to the brown hardboard table top. Both the white instruction card and the white response slip had to be held well to the side, so that they could not be seen directly while the display was being viewed. Similarly, when no dark standard was present in the display, no black could be seen directly while the display was being viewed.

As soon as the student had marked the line, the second investigator changed the gray to judge and then turned over the response slip. The slip carried an identically labeled $100-\mathrm{mm}$ line on the other side. The student was asked to judge the second gray. For all the B groups and for Group W44 in Column 1 of Table 1, the second gray had a reflectance of $7.8 \%$. For the four remaining $W$ groups, the second gray had a reflectance of $44 \%$.

The results of the second judgment were used only to exclude the occasional student who reversed the two ends of the line. For example, the mark corresponding to the gray of $7.8 \%$ reflectance could be further from the dark end of the line than the mark corresponding to the first gray of $19 \%$ reflectance. The $5 \%$ of students who made these reversal errors were replaced.

\section{Results}

Subjective zeros. Figure 1 illustrates the mean judgments listed in Column 6 of Table 1. Panel A shows the derivation of the subjective zero corresponding to black 


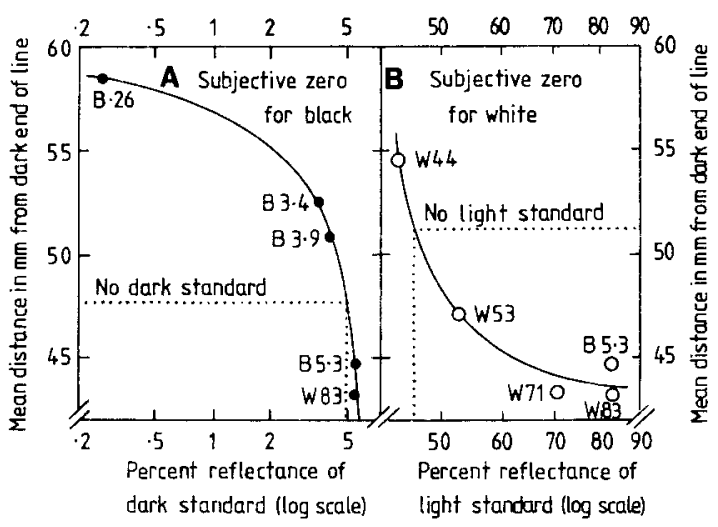

Figure 1. Method for determining the subjective zero. Panel A: Subjective zero corresponding to black in judging lightness when no black is displayed. Panel B: Subjective zero corresponding to white in judging darkness when no white is displayed. See text. The points are labeled to indicate the reflectances of the standards, which are shown also on the abscissa. The labels correspond to the groups in Column 1 of Table 1. W83 has the same reflectance as B5.3.

in judging lightness. The filled points represent conditions with dark standards of different reflectances. The curved function is fitted to the points by eye. The horizontal dotted line represents the mean judgment of Group B-, which has no dark standard. Reading off from the graph, Group B - responds like a group that has a dark standard with a reflectance of $4.9 \%$. Thus, in the absence of a dark standard, the subjective zero corresponding to black in judging lightness is a reflectance of about $5 \%$.

Panel B of Figure 1 shows the derivation of the subjective zero corresponding to white in judging darkness. The unfilled points represent conditions with light standards of different reflectances. As in Panel A, the curved function is fitted to the points by eye. The horizontal dotted line represents the mean judgment of Group W-, which has no light standard. Reading off from the graph, Group W - responds like a group with a light standard of $46 \%$ reflectance. Thus, in the absence of a light standard, the subjective zero corresponding to white in judging darkness is a reflectance of about $45 \%$.
Subjectively equal stimulus spacing for lightness. The last four columns of Table 1 show the predicted mean responses for the four theoretical subjectively equal stimulus spacings. The predictions are derived from Equation 2. In Figure 2, the predicted responses are plotted against the mean observed responses. The groups from which the observed responses are taken are shown on the extreme right of the figure. The broken line sloping up to the right indicates perfect prediction.

None of the predicted response functions in Figure 2 fit the mean observed responses, which fall between the logarithmic and Munsell predictions, but closer to the logarithmic. Table 1 shows that the unfilled points on the logarithmic and Munsell functions are not reliably different from the mean observed responses. The predictions from the square root and linear spacings are all reliably too low.

The square-root prediction closely follows the Munsell prediction, but is always rather smaller. This is due to the $\mathrm{K}$ in Equation 3, which is at its maximum at the $19 \%$ reflectance of the midgray. Thus, changing from the Munsell prediction to the square-root prediction always subtracts more from the numerator of the fraction in Equation 2 than from the denominator. Since the numerator is always smaller than the denominator, the square-root prediction is always smaller than the Munsell prediction. The similar shapes of all the functions in Figure 2 reflect chance differences between the separate groups of students.

Relative stimulus contraction bias. Figure 3 illustrates the distribution of marks on the 100 -mm line for each of the groups of students listed in Table 1 . All 10 groups show a tendency to avoid the middle of the line $(p<.002$ on a binomial test). This is part of the relative stimulus contraction bias (Poulton, 1985, chap. 7), described in the introduction.

Column 5 of Table 1 shows that the reflectance of the dark standard increases in going from Group B.26 through Groups B3.4 and B3.9 to Group B5.3. The filled points in Panels D and B of Figure 3 show that this results in a shift in the largest peaks of the distributions from the

Table 1

Experiment 1: Conditions, Average Responses, and Predictions from Theoretical Subjectively Equal Stimulus Spacings

\begin{tabular}{|c|c|c|c|c|c|c|c|c|c|c|c|}
\hline & & \multicolumn{3}{|c|}{ Percent Reflectance } & \multicolumn{7}{|c|}{ Position of Mark on 100-mm Line (Dark $=0$ ) } \\
\hline \multicolumn{2}{|c|}{ Observers } & \multirow{2}{*}{$\begin{array}{c}\text { Light } \\
\text { Standard } \\
(100 \mathrm{~mm})\end{array}$} & \multirow{2}{*}{$\begin{array}{c}\text { Midgray } \\
\text { to } \\
\text { Judge }\end{array}$} & \multirow{2}{*}{$\begin{array}{c}\text { Dark } \\
\text { Standard } \\
(0 \mathrm{~mm})\end{array}$} & \multicolumn{3}{|c|}{ Observed } & \multicolumn{4}{|c|}{$\begin{array}{c}\text { Predicted if Subjectively } \\
\text { Equal Stimulus Spacing }\end{array}$} \\
\hline Group & $\mathbf{N}$ & & & & Mean & (SE) & Median & Log & Munsell & $\sqrt{\mathrm{Rf}}$ & Linear \\
\hline B.26 & 50 & 83 & 19 & $.26^{*}$ & 58.5 & (1.9) & $62.2 * *$ & 74 & 52 & 45 & 23 \\
\hline B3.4 & 50 & 83 & 19 & 3.4 & 52.4 & $(2.2)$ & $54.5^{* *}$ & $54 \dagger$ & 39 & 35 & 20 \\
\hline B3.9 & 50 & 83 & 19 & 3.9 & 50.9 & (2.4) & $52.3^{* *}$ & $52 \dagger$ & 38 & 33 & 19 \\
\hline B - & 50 & 83 & 19 & & 47.7 & (2.1) & 49.0 & & & & \\
\hline B5. 3 & 52 & 83 & 19 & 5.3 & 44.7 & (2.1) & $38.0^{* *}$ & $46 \dagger$ & 34 & 30 & 18 \\
\hline W83 & 50 & 83 & 19 & 5.3 & 43.2 & (2.4) & $41.0^{* *}$ & $46 \dagger$ & 34 & 30 & 18 \\
\hline W71 & 50 & 71 & 19 & 5.3 & 43.3 & $(2.2)$ & $38.0^{* *}$ & 49 & 37 & 34 & 21 \\
\hline W53 & 50 & 53 & 19 & 5.3 & 47.2 & (2.2) & $42.0^{* *}$ & 55 & $45 \dagger$ & 41 & 29 \\
\hline W- & 50 & & 19 & 5.3 & 51.1 & (2.2) & 49.5 & & & & \\
\hline W44 & 50 & 44 & 19 & 5.3 & 54.5 & (2.5) & $59.5^{* *}$ & 60 & $51 \dagger$ & 48 & 35 \\
\hline
\end{tabular}

*Black rayon velvet. **Median further from 50 than mean. †Prediction not reliably different from mean. 


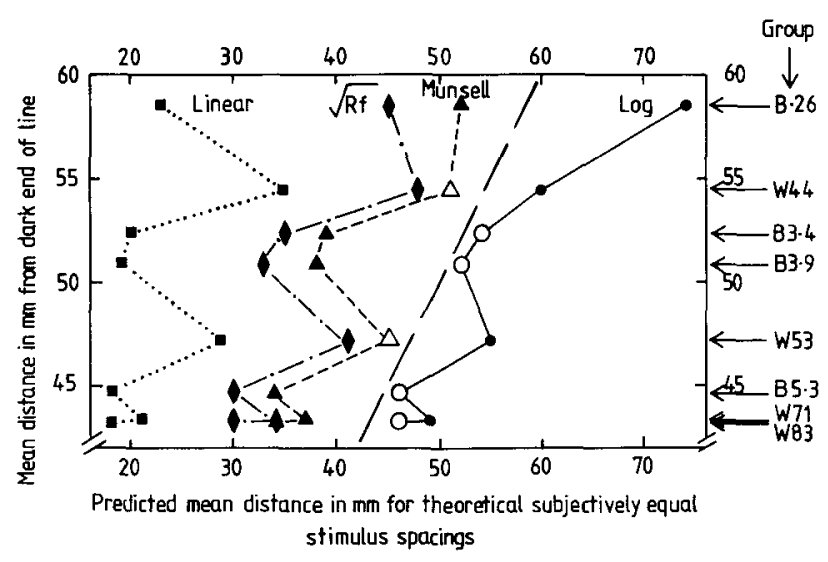

Figure 2. Predictions from four theoretical subjectively equal stimulus spacings for partition judgments of lightness, plotted against the group means of Table 1 . The groups from which the means are taken are indicated on the extreme right of the figure. The sloping broken line represents predictions that would correspond to the group means. The six unfilled points indicate predictions that are not reliably different from the group means. $R f=$ reflectance.

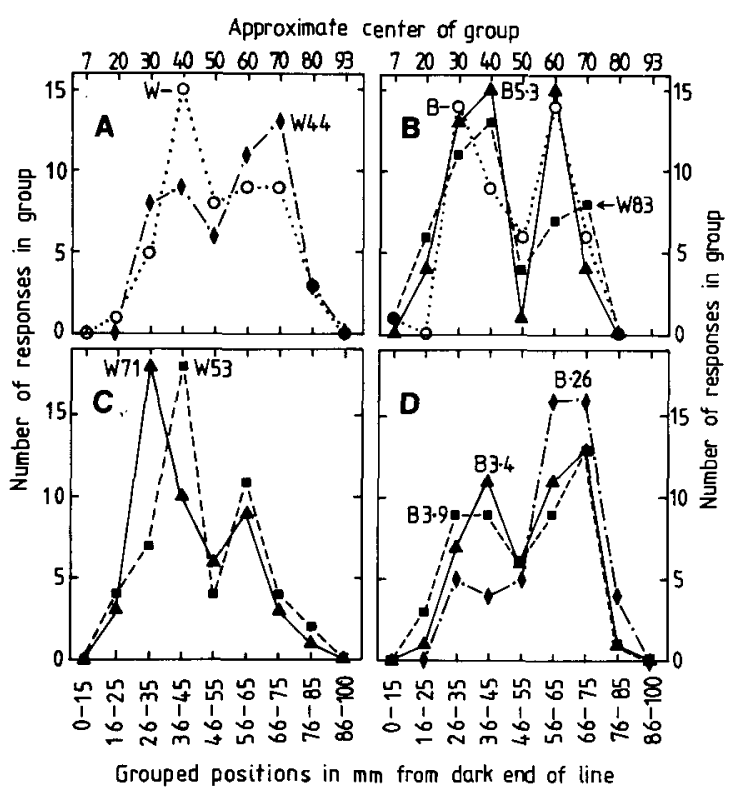

Figure 3. The distributions of the first responses, showing the relative stimulus contraction bias in avoiding the middle of the $100-\mathrm{mm}$ line. The distributions are labeled to correspond to the groups in Column 1 of Table 1.

light side of the $100-\mathrm{mm}$ line to the dark side. But all the peaks avoid the middle of the $100-\mathrm{mm}$ line.

Column 3 of Table 1 shows that the reflectance of the light standard decreases in going from Group W83 through Groups W71 and W53 to Group W44. The filled points in Panels B, C, and A of Figure 3 show that this results in a shift in the largest peaks of the distributions from the dark side of the 100 -mm line back to the light side. But, again, all the peaks avoid the middle of the 100 mm line.

For half the students in each group, the darkest gray in the display is on the left. For the other half, the darkest gray is on the right. The labeling of the two ends of the line always corresponds to the arrangement of the grays in the display. Thus, whether the average students deviate to the left or to the right in avoiding the middle of the line, the bias should balance out for the group means. The bias should not affect the means of the distributions in Figure 3, unless the students deviate systematically towards the dark or light end of the line.

However, the bias does affect the medians of the eight groups that made partition judgments with two standards. For the eight groups, Columns 6 and 8 of Table 1 show that the median is always further from 50 than is the mean ( $p<.01$ on a binomial test). Even when the Groups Band $\mathrm{W}-$ are added, the tendency for the median to be further from 50 than the mean is still reliable on the twotailed Wilcoxon test $(\mathrm{p}<.02)$.

The relation between the median and mean is modeled in Figure 4. The model shows that avoiding the middle of the $100-\mathrm{mm}$ line moves some of the responses from one side to the other side of the center of the unbiased distribution of responses, as indicated by the vertical dashed line. This shifts the median of the new distribution of responses in a direction away from the middle of the 100-mm line. Thus, the biased median should lie further from the middle of the $100-\mathrm{mm}$ line than does the mean, as for the eight groups in Table 1 that made partition judgments with two standards. Since the median is biased, whereas the mean should not be biased, the mean is the measure that is used throughout Experiment 1.

The relative stimulus contraction bias does not appear to affect the second response. Presumably, the students then use their first response as an additional reference. They no longer use the middle of the line as the key reference, as they presumably do for the first response.

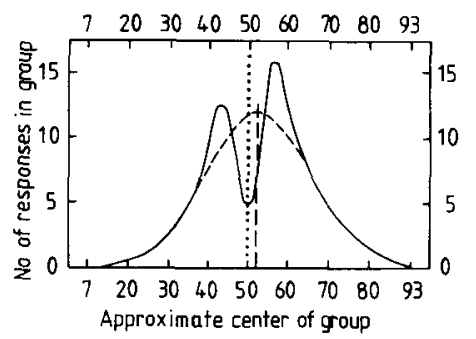

Figure 4. Model for the relative stimulus contraction bias in marking a 100-mm line for the first time. The symmetric unimodal distribution represents unbiased responses. The center of the distribution of responses is indicated by the vertical dashed line. The center lies just above the middle of the $100-\mathrm{mm}$ line, which is indicated by the vertical dotted line. When this is so, avoiding the middle of the line produces the asymmetric bimodal distribution represented by the solid function. This biases the median, but need not bias the mean. See text. 


\section{EXPERIMENT 2}

Experiment 2 was designed to discover whether the subjective zeros found for the first partition judgments with a single standard applied to the first unbiased fractional numerical judgments of lightness and darkness. The two kinds of judgment were compared, using identical displays. Provided the observers used numbers linearly for the first fractional numerical judgments, the results should be comparable. If so, the subjective zeros for the two kinds of judgment can be derived using the same method, and should have the same values within the limits of the experimental variability.

The experimental design is given on the left side of Table 2 . The responses of Groups B- and $\mathbf{W}-$ are compared with the responses of similar groups of uninitiated students who made their first fractional numerical judgment of lightness or darkness with the standard called 100 .

\section{Subjective Zero for Black in the First Fractional Numerical Judgment of Lightness}

The display for Group $\mathrm{Bn}$ in Row 2 of Table 2 was the same as the display for Group B- in Row 1. The only differences were in the kind of responses and in the instructions. For Group Bn, the instructions ran: "You will see two pieces of paper lying side by side, one white and one gray. If the lightness of the white paper is called 100 , what (smaller) number describes the lightness of the gray paper? Please write down your answer on the slip of paper provided." The slip of white paper measured $10 \times 8 \mathrm{~cm}$.

Table 2 shows that the mean response of Group $\mathrm{Bn}$ is 45.2 (SE 2.5), compared with the mean response of Group B - of 47.7 (2.1). The difference of 2.5 lies well within 2 standard errors of the difference, 6.5 . The medians are also fairly comparable, 45.0 and 49.0 , respectively. This suggests that the students used numbers linearly in making their first fractional numerical judgment of lightness. The agreement suggests also that the students used a subjective zero of about the same size as that used for the partition judgments.

It should be possible to use the results illustrated in Figure 1A to determine the size of the subjective zero for black that was used by Group Bn. If so, the mean of 45.2 indicates a subjective zero corresponding to black of $5.3 \%$ reflectance. This can be compared with the subjective zero of $4.9 \%$ reflectance, which is derived from the mean of
Group B - . The difference of .4 percentage points is relatively small.

\section{Subjective Zero for White in the First Fractional Numerical Judgment of Darkness}

The bottom part of Table 2 shows a similar comparison between the average responses of Group Wn and the comparable responses of Group W-. Again, the display is the same for the two groups. The only differences are in the responses and in the instructions. The instructions for Group Wn correspond to the instructions for Group $\mathrm{Bn}$, but with dark gray, less dark, and darkness substituted for white, gray, and lightness.

In Table 2, the average responses of Group Wn are subtracted from 100 . This corresponds to the dark gray standard being scored as 0 , as for Group W- and for all the other groups in Table 1 . Table 2 shows that 100 minus the mean response of Group Wn is 54.5 (SE 2.4); for Group W- it is 51.1 (2.2). The difference of 3.4 lies well within 2 standard errors of the difference, 6.5 . The medians are still more comparable, 50.0 and 49.5 , respectively. This suggests that the students used numbers linearly in making their first fractional numerical judgment of darkness, and that they used a subjective zero of about the same size as that used for the partition judgments.

It should be possible to use the results illustrated in Figure 1B to determine the size of the subjective zero for white that was used by Group Wn. If so, the mean of 54.5 indicates a subjective zero corresponding to white of $44 \%$ reflectance. This can be compared with the subjective zero of $46 \%$ for Group $\mathrm{W}-$. The difference of 2 percentage points is relatively small.

Two conclusions follow from these results. First, the fractional numerical judgments of both lightness and darkness use subjective zeros. Second, the subjective zeros are of about the same size as the subjective zeros found for the partition judgments.

\section{DISCUSSION}

\section{Exponents Corrected for the Subjective Zero}

The results in Table 2 suggest that in making a first fractional numerical judgment of lightness or darkness, the students use a subjective zero that is well above the absolute threshold. If this is so, the corresponding power func-

Table 2

Experiment 2: Comparisons Between Partitioning on a Line and Fractional Numerical Judgments

\begin{tabular}{|c|c|c|c|c|c|c|c|c|}
\hline \multicolumn{2}{|c|}{ Observers } & \multicolumn{2}{|c|}{ Percent Reflectance } & \multicolumn{4}{|l|}{ Response (Dark $=0$ ) } & \multirow{2}{*}{$\begin{array}{l}\text { Percent Reflectance } \\
\text { of Subjective Zero }\end{array}$} \\
\hline Group & $\mathbf{N}$ & Standard & Midgray & Kind & Mean & (SE) & Median & \\
\hline \multicolumn{9}{|c|}{ Subjective Zero for Black } \\
\hline $\begin{array}{l}\text { B- } \\
\mathrm{Bn}\end{array}$ & $\begin{array}{l}50 \\
50\end{array}$ & $\begin{array}{l}83 \\
83\end{array}$ & $\begin{array}{l}19 \\
19\end{array}$ & $\begin{array}{l}\text { Partition a line between white and black } \\
\text { Fractional numerical judgment with white }=100\end{array}$ & $\begin{array}{l}47.7 \\
45.2\end{array}$ & $\begin{array}{l}(2.1) \\
(2.5)\end{array}$ & $\begin{array}{l}49.0 \\
45.0\end{array}$ & $\begin{array}{l}4.9 \\
5.3\end{array}$ \\
\hline \multicolumn{9}{|c|}{ Subjective Zero for White } \\
\hline $\begin{array}{l}\text { W- } \\
\text { Wn }\end{array}$ & $\begin{array}{l}50 \\
50\end{array}$ & $\begin{array}{l}5.3 \\
5.3\end{array}$ & $\begin{array}{l}19 \\
19\end{array}$ & $\begin{array}{l}\text { Partition a line between dark gray and white } \\
\text { Fractional numerical judgment with dark gray }=100\end{array}$ & $\begin{array}{l}51.1 \\
54.5^{*}\end{array}$ & $\begin{array}{l}(2.2) \\
(2.4)\end{array}$ & $\begin{array}{l}49.5 \\
50.0\end{array}$ & $\begin{array}{l}46 \\
44\end{array}$ \\
\hline
\end{tabular}

${ }^{*}$ Mean subtracted from 100 , to make dark $=0$. 
tions for lightness and darkness should contain a constant $S_{0}$ corresponding to the subjective zero. The $S_{0}$ is required to describe more precisely what students do when they judge stimulus magnitudes. It should be used whether or not it improves the fit of the power function, as Ekman $(1958,1959,1961)$ suggests it may.

Stevens and Galanter's (1957, Figure 12A circles) median numerical magnitude judgments of lightness are approximately linear when plotted against reflected brightness on a $\log \log$ plot. Figure 2 shows that the subjectively equal stimulus spacing for judgments of lightness is fairly close to logarithmic. Thus, Stevens and Galanter's median students must have used numbers either as ratios or with a logarithmic response bias.

The subjective zero can be determined by replotting the results on a linear plot and extrapolating the curved function to the point at which the subjective judgment would be zero. For the first investigation that Stevens and Galanter mention, the method gives a subjective zero that is about $1.2 \%$ of the reflectance of the lightest stimulus. Correcting for the subjective zero reduces the corresponding exponent for lightness from 1.3 to 1.15 , a reduction of $12 \%$.

\section{Using Numbers Linearly or as Ratios}

It was pointed out in the last section that, in judging lightness, Stevens and Galanter's median students used numbers either as ratios or with a logarithmic response bias. This is presumably because Stevens $(1956$, p. 3 ) instructed his students to use numbers as ratios. By telling his observers to use numbers as ratios, Torgerson (1960, p. 27) obtained an exponent for lightness "very nearly" the same size as Stevens and Galanter's, whereas Table 2 shows that the average fractional numerical judgments of Group $\mathrm{Bn}$ are similar to the average partition judgments of Group B-. This indicates that the average students in Group Bn used numbers linearly.

There are two influences whose absence encouraged the students in Groups Bn and Wn to use numbers linearly. First, the instructions for the numerical magnitude judgments did not follow Stevens's instructions to use numbers as ratios. Second, of the 100 students in the two groups that made numerical magnitude judgments, 99 used numbers between 75 and 10 . Only one student used a number less than 10. For the 99 students, there is no step change in the number of digits used as responses, which can produce a logarithmic response bias in the use of numbers (Banks \& Hill, 1974; see Poulton, 1979, p. 783). The absence of ratio instructions, and of a step change in the number of digits used as responses, encouraged the observers to use numbers linearly.

The agreement between the means of Groups B - and $\mathrm{Bn}$ in the top part of Table 2 can be contrasted with the disagreement in Table 1 of Warren and Poulton (1960). Warren and Poulton found a mean first linear response for lightness of 38.1 (SE 2.2) and a comparable mean first fractional numerical response of 28.8 (2.0). The differ- ence of 9.3 is reliable $(p<.01)$. Yet the procedures used in the investigations of the top part of Table 2 follow the procedures used by Warren and Poulton.

The key difference is that Warren and Poulton used a dark gray to judge, with a reflectance of $7.8 \%$. In first partitioning a line, the dark gray introduces the second part of the relative stimulus contraction bias, which is described in the introduction.

The students avoided the end of the line labeled black. Of the $\mathbf{5 0}$ first partition judgments, the judgment that lies closest to the black end of the line is $17 \mathrm{~mm}$ away, whereas 12 of the 50 first fractional numerical judgments are 17 or less. This is responsible for the reliable difference between Warren and Poulton's two means.

The relative stimulus contraction bias does not affect the medians. The difference between Warren and Poulton's (1960, Table 1) first medians is only 4 . The difference is in the same direction and of the same size as the difference between the medians in the top part of Table 2 .

\section{Nearly Logarithmic Subjectively Equal \\ Stimulus Spacing}

Figure 2 shows that the subjectively equal stimulus spacing for the very first partition judgments of lightness is nearest to logarithmic. The logarithmic prediction fits the mean responses better than does the Munsell scale of neutral value.

The largest discrepancy from the logarithmic prediction is shown by Group B.26 at the top of Figure 2. The predicted response of 74 is produced by the black rayon velvet standard, which has a reflectance of only $.26 \%$. Column 9 of Table 1 shows that the 74 is over twice as far from 50 as is the next most extreme logarithmic prediction of 60 . Figure 3 shows that 74 lies in the upper tail of all the distributions of responses. For Group B.26 in Panel D, there are only five responses of 74 or greater, the highest being 78 .

The discrepancy between the predicted 74 and the observed mean of 58.5 is presumably exaggerated by the response contraction bias, or regression effect (Poulton, 1979, p. 789; Stevens \& Greenbaum, 1966). The students mark the line closer to the middle than they should. The bias is greatest for the predicted value of 74 , because 74 lies furthest from the middle of the line.

The Munsell scale of neutral value would not be expected to agree well with the subjectively equal stimulus spacing found in the present investigation, for two reasons. First, Newhall et al. (1943) used practiced observers, whose judgments could be biased by transfer from prior series of judgments. Second, Newhall et al. used small patches of gray paper. Although it does not appear to be mentioned anywhere, the small patches seem, from the photographs (Newhall, 1940, Figure 4), to be about $12 \times 18 \mathrm{~mm}$ and to be spaced about $3 \mathrm{~mm}$ apart on their white, gray, or black backgrounds. With these small patches, the judgments of relative lightness depend on the reflectance of the background (Newhall et al., 1943, 
Figure 14). The background chosen for the Munsell scale of neutral value has a reflectance of $20 \%$.

In contrast, for the large patches of $28 \times 15 \mathrm{~cm}$ used in the present investigation with their long sides contiguous, the reflectance of the background does not appreciably affect the judgments of relative lightness (Warren \& Poulton, 1960, Experiments 1 and 3). Thus, with their practiced observers and small gray patches, Newhall et al.'s Munsell scale of neutral value would not be expected to agree well with the subjectively equal stimulus spacing obtained from the first judgments of the present investigation.

\section{Units of Lightness and Darkness are of About Equal Size}

The previous section shows that the subjectively equal stimulus spacing is fairly close to logarithmic when Group B.26, with its black rayon velvet, is excluded. Table 2 shows that the students in Groups Bn and Wn used numbers linearly in making their fractional numerical judgments. Taking these two results together, it follows that the fractional numerical judgments of Groups $B n$ and $\mathrm{Wn}$ should be related more or less linearly to the ratios of the reflectances. Thus, it should be possible to compare the fractional numerical judgments of the two groups in logarithmic units of reflectance ratio, once the reflectances are corrected for the subjective zeros. This is equivalent to comparing the sizes of exponents when numbers are used as ratios.

For Group Bn in Table 2, when the reflectances judged are corrected for the subjective zero, their ratio is:

$$
\frac{83-5.3}{19-5.3}=\frac{77.7}{13.7}=5.68, \text { or } .754 \log \text { units. }
$$

For Group Wn, correcting for the subjective zero makes $\left(\mathrm{S}-\mathrm{S}_{0}\right)$ a minus quantity. The difficulty can be overcome by using the reciprocals of the reflectances. With the approximately logarithmic subjectively equal stimulus spacing, taking reciprocals simply gives the reciprocal of the ratio of the reflectances. This can be corrected by calculating its reciprocal. The appropriateness of taking reciprocals is indicated by Torgerson's (1960, Figure 3.6) finding that subjective darkness is the reciprocal of subjective lightness when ratio judgments are made. Twice as dark means half as light.

For Group Wn, when the reflectances judged are corrected for the subjective zero, their ratio is:

$$
\begin{aligned}
\frac{1 / 5.3-1 / 44}{1 / 19-1 / 44} & =\frac{.189-.0227}{.0526-.0227}=\frac{.1663}{.0299} \\
& =5.56 \text { or } .745 \log \text { units. }
\end{aligned}
$$

This is almost the same size in log units as the .754 of Group $\mathrm{Bn}$. The agreement makes it possible to compare directly the size of the units of lightness used by Group Bn with the size of units of darkness used by Group Wn.
Group Bn judged lightness, so the larger of the two reflectances is called 100 . Table 2 shows that the smaller reflectance received a mean judgment of 45.2. The difference in units of lightness is 54.8. Thus, one unit of lightness corresponds to a reflectance ratio of:

$$
.754 / 54.8=.0138 \log \text { units. }
$$

Group Wn judged darkness, so the smaller of the two reflectances is called 100 . Here Table 2 shows that the mean difference in units of darkness is 54.5. Thus, the unit for darkness corresponds to a reflectance ratio of:

$$
.745 / 54.5=.0137 \log \text { units. }
$$

This value is almost the same as the value of $.0138 \mathrm{log}$ units, which corresponds to one unit of lightness. Thus, when corrected for the subjective zero, one unit of lightness corresponds to about the same reflectance ratio as one unit of darkness. If the students were to use numbers as ratios, the equivalent finding would be that the exponent for lightness was the same size as the exponent for darkness.

\section{When to Use and Not to Use Medians}

With magnitude judgments using numbers, the investigator may be uncertain whether observers used numbers linearly or with a logarithmic response bias. If so, the investigator should use the median response, because medians are not affected by monotonic transformations such as the logarithmic response bias. The middle response of a group remains the middle response. Thus, for example, in comparing the judgments of Groups Bn and Wn in Table 2 with the judgments of other groups, the median would be the appropriate statistic to use.

However, when observers respond by marking a line, their responses should not be affected by the logarithmic response bias in the use of numbers. Thus, medians have no advantage over arithmetic means. Experiment 1 shows that medians should not be used for the first judgments of uninitiated observers, since the medians are likely to be biased by the tendency to avoid the middle of the line. Thus, here, arithmetic means should be used, provided they are unbiased. For example, in comparing the judgments of Groups B - and W - in Table 2 with the judgments of other groups, the arithmetic mean would be the appropriate statistic to use.

Having to use medians for Groups Bn and Wn but arithmetic means for Groups B - and W- could cause problems in comparisons of Groups Bn and Wn with Groups B- and W-. Fortunately, there are no such problems in Table 2 . First, as has already been pointed out, the arithmetic means of Groups Bn and Wn are not affected by the logarithmic response bias in the use of numbers.

Second, the medians of Groups B - and W - are not affected by the relative stimulus contraction bias, which is modeled in Figure 4. Table 2 shows that both me- 
dians are closer to 50 than are the corresponding means. This is the opposite direction to the direction produced by the relative stimulus contraction bias. Thus, both the arithmetic means and the medians can be used to compare Group Bn with Group B-, and Group Wn with Group W-, as is done in discussing the results of Table 2.

There is a compromise method between fractional numerical judgments and marking a line. The students mark a line labeled 100 at one end and 0 at the other. The method was used by Poulton et al. (1968). It cannot be recommended, however, because the students have a choice of two strategies. They can mark the line, paying no attention to the numbers 100 and 0 at the two ends. If so, the first judgment is likely to be biased by an avoidance of the middle of the line, as in the model of Figure 4. Thus, the average judgment should not be represented by the median.

The second strategy is first to pick a number between 100 and 0 and then to mark the line at the appropriate position. Suppose the students adopt this second strategy. Their responses may then show a logarithmic response bias, from the choice of both one-digit and two-digit numbers. If so, their average judgment should not be represented by an arithmetic mean.

When some students in a group use one strategy and some use the other strategy, neither the median nor the arithmetic mean is likely to provide an unbiased measure of the average first response. Thus, the method should not be used to obtain average first judgments from uninitiated students.

\section{REFERENCES}

BANKs, W. P., \& HILL, D. K. (1974). The apparent magnitude of number scaled by random production. Joumal of Experimental Psychology, 102, 353-376.

BiRNBAUM, M. H. (1978). Differences and ratios in psychological measurement. In N. J. Castellan \& F. Restle (Eds.), Cognitive theory (Vol. 3). Hillsdale, NJ: Erlbaum.

BirnbaUM, M. H. (1980). Comparison of two theories of "ratio" and "difference" judgments. Joumal of Experimental Psychology: General, 109, 304-319.

ExMAN, G. (1958). Two generalized ratio scaling methods. Joumal of Psychology, 45, 287-295.

EkmaN, G. (1959). Weber's law and related functions. Journal of Psyhology, 47, 343-352.

ExMAN, G. (1961). A simple method for fitting psychophysical power functions. Journal of Psychology, 51, 343-350.
Fagot, R. F., Eskildsen, P. R., \& Stewart, M. R. (1966). Effects of rate of change in physical intensity on bisection and fractionation judgments of brightness. Journal of Experimental Psychology, 72, 880-886.

LoNgmore, J., \& Petherbridge, P. (1961). Munsell value/surface reflectance relationships. Joumal of the Optical Society of America, 51, 370-371.

NewhalL, S. M. (1940). Preliminary report of the O.S.A. subcommittee on the spacing of the Munsell colors. Journal of the Optical Society of America, 30, 617-645.

Newhall, S. M., Nickerson, D., \& Judd, D. B. (1943). Final report of the O.S.A. subcommittee on the spacing of the Munsell Colors. Journal of the Optical Society of America, 33, 385-418.

Poulton, E. C. (1968). The new psychophysics: Six models for magnitude estimation. Psychological Bulletin, 69, 1-19.

Poulton, E. C. (1975). Range effects in experiments on people. American Journal of Psychology, 88, 3-32.

Poulton, E. C. (1979). Models for biases in judging sensory magnitude. Psychological Bulletin, 86, 777-803.

Poulton, E. C. (1982). Influential companions: Effects of one strategy on another in the within-subjects designs of cognitive psychology. Psychological Bulletin, 91, 673-690.

Poulton, E. C. (1985). Bias in judgment. San Francisco: Academic Press.

Poulton, E. C., Simmonds, D. C. V., \& Warren, R. M. (1968). Response bias in very first judgments of the reflectance of grays: Numerical versus linear estimates. Perception \& Psychophysics, 3, 112-114.

STEVENS, S. S. (1956). The direct estimate of sensory magnitudesloudness. American Journal of Psychology, 69, 1-25.

Stevens, S. S. (1975). Psychophysics: Introduction to its perceptual, neural, and social prospects. New York: Wiley.

STEVENS, S. S., \& GALANTER, E. H. (1957). Ratio scales and category scales for a dozen perceptual continua. Journal of Experimental Psychology, 54, 377-411.

Stevens, S. S., \& Greenbaum, H. B. (1966). Regression effect in psychophysical judgment. Perception \& Psychophysics, 1, 439-446.

TAYLOR, M. M. (1961). Effect of anchoring and distance perception on the reproduction of forms. Perceptual and Motor Skills, 12, 203-230.

Teghtsoonian, R. (1971). On the exponents in Stevens' law and the constant in Ekman's law. Psychological Review, 78, 71-80.

Torgerson, W. S. (1960). Quantitative judgment scales. In H. Gulliksen \& S. Messick (Eds.), Psychological scaling: Theory and applications. New York: Wiley.

WARREN, R. M. (1958). A basis for judgments of sensory intensity. American Journal of Psychology, 71, 675-687.

WARREN, R. M. (1969). Visual intensity judgments: An empirical rule and a theory. Psychological Review, 76, 16-30.

WARREN, R. M. (1981). Measurement of sensory intensity. Behavioral and Brain Sciences, 4, 175-189.

Warren, R. M., \& Poulton, E. C. (1960). Basis for lightnessjudgments of grays. American Journal of Psychology, 73, 380-387.

(Manuscript received August 15, 1984; revision accepted for publication March 26, 1985.) 